\title{
Sciendo
}

\section{FUNCTIONS OF CIRCULAR RNAS INVOLVED IN ANIMAL SKELETAL MUSCLE DEVELOPMENT - A REVIEW}

\author{
Patricia Adu-Asiamah, Qiying Leng, Haidong Xu, Jiahui Zheng, Zhihui Zhao, Lilong An, Li Zhang* \\ College of Agriculture, Guangdong Ocean University, Zhanjiang 524088, P.R. China \\ •Corresponding author: zhangli761101@163.com
}

\begin{abstract}
Circular RNAs (circRNAs) have been identified in the skeletal muscle of numerous species of animals. Their abundance, diversity, and their dynamic expression patterns have been revealed in various developmental stages and physiological conditions in skeletal muscles. Recently, studies have made known that circRNAs widely participate in muscle cell proliferation and differentiation. They are also involved in other life processes such as functioning as microRNA (miRNA) sponges, regulators of splicing and transcription, and modifiers of parental gene expression with emerging pieces of evidence indicating a high chance of playing a vital role in several cells and tissues, especially the muscles. Other research has emphatically stated that the growth and development of skeletal muscle are regulated by proteins as well as non-coding RNAs, which involve circRNAs. Therefore, circRNAs have been considered significant biological regulators for understanding the molecular mechanisms of myoblasts. Here, we discuss how circRNAs are abundantly expressed in muscle (myoblast) and their critical roles in growth and development.
\end{abstract}

Key words: circRNAs, skeletal muscle, growth, myoblast, development

According to the statistics of the Food and Agriculture Organization (FAO), overall world meat production increased to more than 336 million metric tons in 2018 (Conway, 2018). For several decades, genetic improvements in animal production have improved growth rate and body size, resulting in a higher yield of meat in animals. Skeletal muscle makes up approximately $40 \%$ of the body's mass, and it is an essential component of food from farm animal species, which directly relates to meat quantity and quality. Also, the skeletal muscle exhibits a wide diversity of shapes, sizes, anatomical locations, and physiological functions. Myogenesis is a complex process encompassing multiple steps and regulation factors. Myofibre ontogenesis also begins very early during embryonic life with the total number of fibres fixed during foetal life. The skeletal muscle mass is generally determined by a balance between muscle protein synthesis and degradation. It has been noted that muscle 
development defines muscle mass, muscle fiber type, and some neurotic muscle changes. Also, the growth and development of skeletal muscle involve a series of very complex biological processes, controlled by many signaling pathways, genes, transcription factors and ncRNA (Bassel-Duby and Olson, 2006; Schiaffino et al., 2007; Luo et al., 2013; Nie et al., 2015). The recent identification of circular RNAs (circRNAs) has opened up a new field of investigation to understand the molecular processes which control muscle development. This review will highlight our present understanding of skeletal muscle circRNA regulation and how circRNAs may control skeletal muscle development, function, and adaptation.

\section{CircRNAs highly enriched in muscle}

Circular RNAs (circRNAs) are classes of non-coding RNA which were found decades ago in plants as viral genomics (Nitsche et al., 2014) and later in eukaryotes. circRNAs are generally made by a specific mode of alternative splicing, termed back-splicing, activated by the spliceosomal mechanism (Vicens and Westhof, 2014; Lasda and Parker, 2014; Chen and Yang, 2015). They are stable molecules which are highly abundant in skeletal muscle with large numbers recently found in human (Salzman et al., 2013; Rybak-Wolf et al., 2014), monkey (Abdelmohsen et al., 2015), pig (Liang et al., 2017), chicken (Ouyang et al., 2017), mouse (Memczak et al., 2013), nematode (Ivanov et al., 2015), zebrafish and coelacanth (Nitsche et al., 2014). They are often shown in tissue at different developmental stage-specific expression patterns. Some biological functions of circRNAs in muscle have been discovered, including their involvement in muscle development and the regulation of gene expression at multiple levels. The summary of the circRNAs involved in the progress of skeletal muscle in the different species is illustrated in Table 1 below.

\section{CircRNAs involved in myoblast proliferation and differentiation}

Until now, a total of 8 circRNAs have been found involved in myoblast proliferation and differentiation (Figure 1). Examples are circFGFR4 (Li et al., 2018 a), circFUT10 (Li et al., 2018 b), circ-ZNF609 (Legnini et al., 2017), circRBFOX2 (Ouyang et al., 2017) and so on. Seven of them functioned as miRNA sponges to regulate the target genes during myoblast proliferation and differentiation. For instance, circFGFR4 alters Wnt3a expression by competing for miR-107 as a ceRNA to regulate myogenic differentiation. Similarly, circFUT10 in bovine muscle cell was confirmed to increase differentiation and decrease the proliferation of myoblasts by directly binding to miR-133a and inhibiting miR-133a activity ( $\mathrm{Li}$ et al., $2018 \mathrm{~b}$ ). Besides, circLMO7 was revealed to protect muscle cell from apoptosis (cell survival), promote cell proliferation, and decrease cell differentiation by sponging miR-378a-3p in bovine myoblasts (Wei et al., 2017). These researches make it clear that circRNAs play a potential regulatory role in myogenesis by the sequestration of miRNAs. While circ-ZNF609 (Legnini et al., 2017) consists of direct reading scaffolds, which can translate and regulate myoblast differentiation and proliferation. Another notable circRNA is circMbl, which arises from MBL pre-mRNA, demonstrating that MBL could bind to its pre-mRNA and affect myoblast differentiation and proliferation (Ashwal-Fluss et al., 2014). 


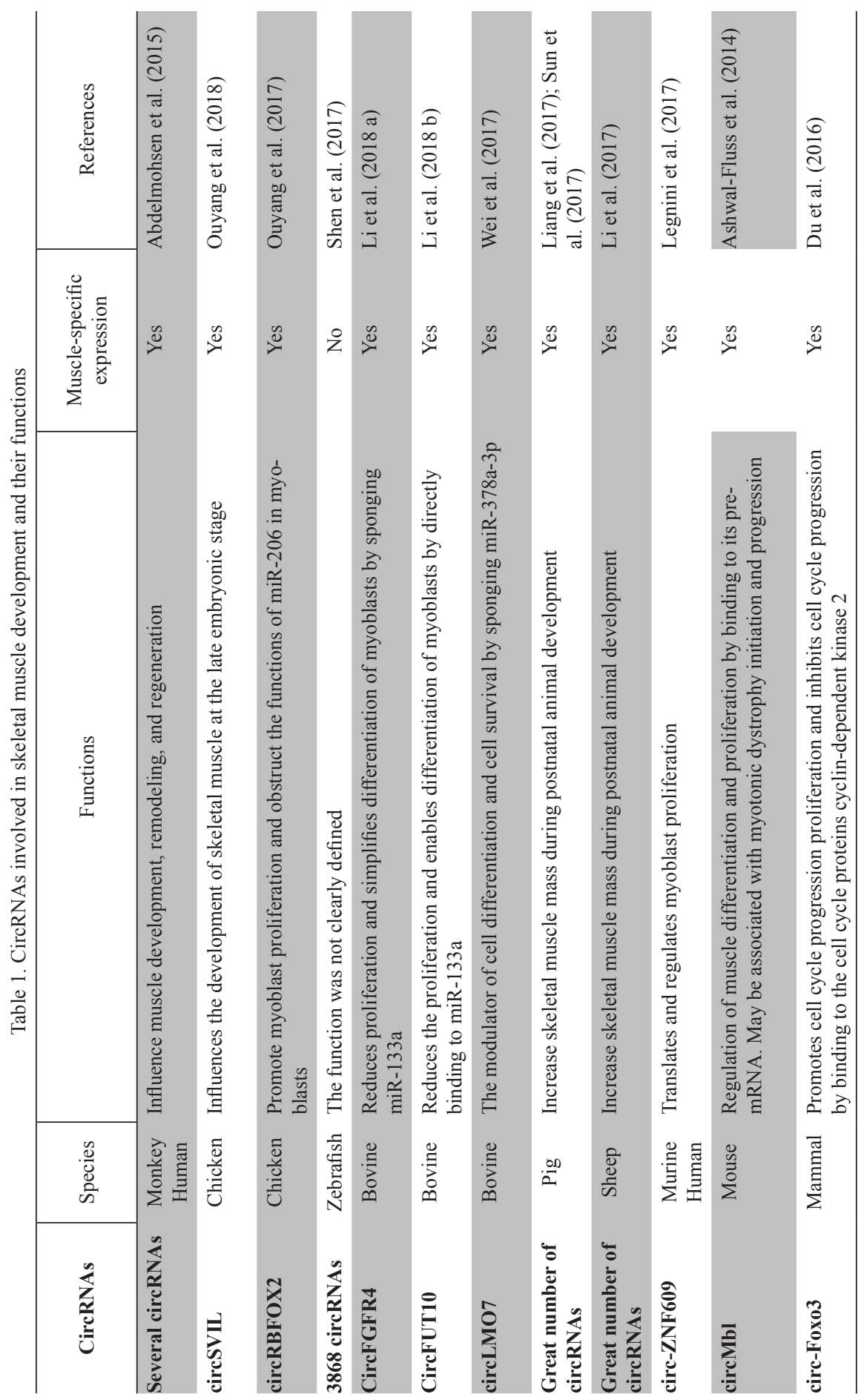




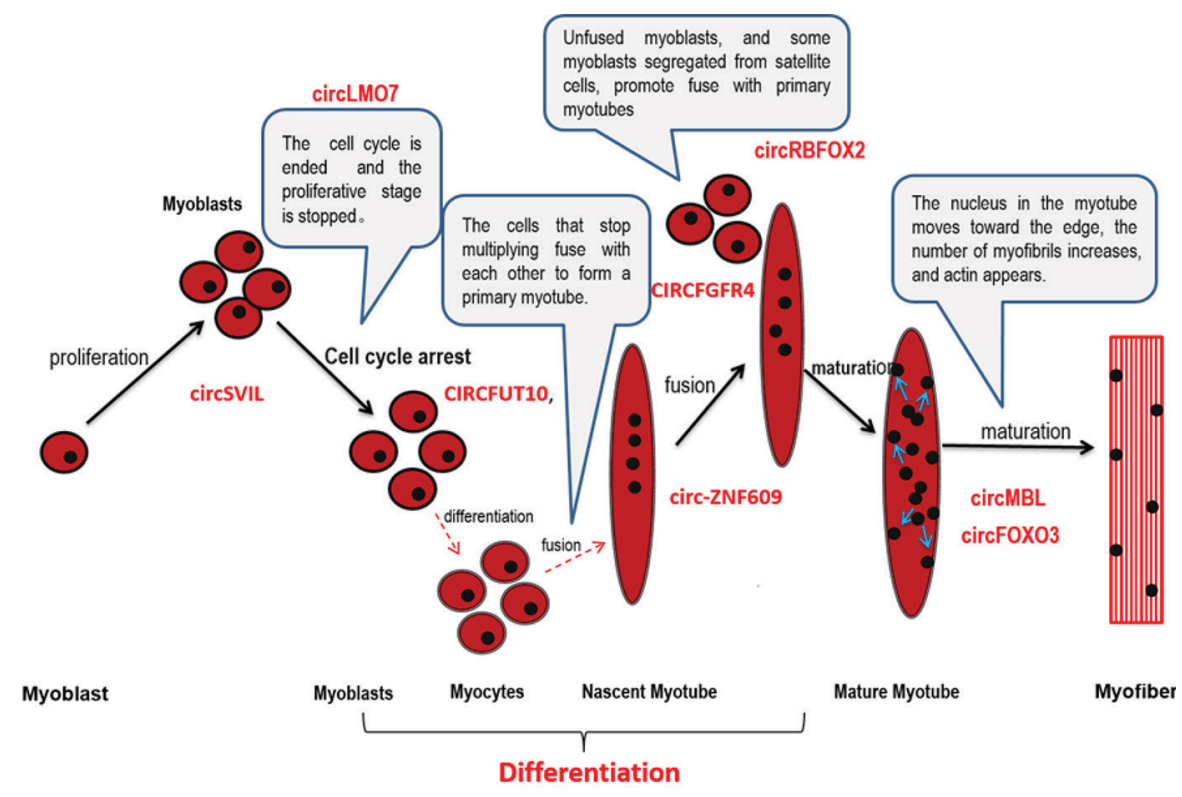

Figure 1. Illustration of circRNAs acting as miRNAs sponge in skeletal muscle growth at various developmental stages

\section{CircRNAs involved in skeletal muscle development during aging}

CircRNAs are abundantly expressed and have a potential function on the development of muscle. Generally, CircRNAs can influence muscle physiology, including muscle development, remodeling, and regeneration. Abdelmohsen et al. (2015) identified the abundance of circRNAs expressed in the skeletal muscles of the monkey. From the experiment, some of the muscle circRNAs identified may directly or indirectly interrupt the expression levels, localization, and function of myogenic transcription factors, myogenic RBPs, or myomiRs (muscle microRNAs).

Moreover, it was established systematically by Abdelmohsen et al. (2015) that the circRNAs in skeletal muscle of monkeys changed with advancing age, indicating that circRNAs have a potential function on age-related muscle disorders. Also, it was disclosed that circRNAs are involved in chronic CD28-associated CD8 (+) T cell aging at the cellular level (Abdelmohsen et al., 2015). A study on chicken embryo skeletal muscle has shown that circRNAs may affect muscle growth (Ouyang et al., 2018). It has been unveiled that the expression form of circSVIL increases conserved and influenced the development of skeletal muscle at the late embryonic stage. Active circRNAs regularly display tissue in developmental stage-specific expression forms (Salzman et al., 2013; Venø et al., 2015; Westholm et al., 2014). Another example reveals circRNAs' ability to increase skeletal muscle mass during postnatal animal development. Previously, it was shown that proliferation and fusion of satellite cells could lead to a rise in the number of myonuclei, which control muscle growth during the initial stages of postnatal development (Schiaffino et al., 2013). 
The experiment of Li et al. (2017) showed that the sheep muscle has a significant number of circRNAs which were differentially shown by screening both prenatal and postnatal muscle of sheep. Similar results were also found in pigs study (Liang et al., 2017). The circRNA expression profiles of the longissimus dorsi muscle were also given to simplify the better understanding of the roles circRNAs play in the progress of muscle.

\section{Roles of circRNA in skeletal muscle disorders}

Various studies have demonstrated the functions of circRNA in muscle development, including adverse functions too. Recent researches prove that circRNAs profoundly influence the physiology and pathology of skeletal muscle. An exciting study observed that the expression of circRNAs in skeletal muscle of monkey changed with evolving age, showing that circRNAs may have a possible function on age-related muscle disorders (Abdelmohsen et al., 2015). Also, another study indicated that circRNAs are controlled during murine and human muscle differentiation and whose expression is transformed in Duchenne muscular dystrophy (DMD) myoblasts, especially circ-QKI and circ-ZNF609 (Legnini et al., 2017).

Another interesting circRNA is circMbl. Findings confirmed that MBL could bind to its pre-mRNA and encouraged circMbl production. CircMbl might be associated with myotonic dystrophy initiation and progression (Ashwal-Fluss et al., 2014). MBL can regulate MBL pre-mRNA splicing effectiveness via the action of both MBL mRNA and circMbl. Additionally, circMbl can act as an MBL sponge. Functional deficiency of MBL leads to a severe progressive disorder known as myotonic dystrophy. Hence, circMbl could be involved in myotonic dystrophy initiation and progression (Ashwal-Fluss et al., 2014). Furthermore, Circ-Foxo3 was identified to be highly expressed in non-cancer cells which inhibited cell cycle progression by binding to the cell cycle proteins cyclin-dependent kinase 2 (cell division protein kinase 2 or CDK2) and cyclin-dependent kinase inhibitor 1 (or p21), resulting in the formation of a ternary complex.

\section{Mechanism of circRNAs in regulating muscle development}

In current reports, the primary mechanism of circRNAs in muscle development is the miRNA sponge, which acts through the circRNA-miRNA-mRNA regulatory networks. Most findings have predicted that circRNAs could regulate the miRNA expression by competitive binding the target gene in muscle cells. CircRNAs form molecular sponges to absorb and isolate miRNAs and can deactivate miRNA-mediated degradation of mRNAs (Kulcheski et al., 2016). For instance, circSVIL proved to promote the development of skeletal muscle at the late embryonic stage carries four binding sites for miR-203 and was recently reported to interact with miR-203 by a dual-luciferase reporter and RNA pull-down assays as well as to increase the expression of miR-203 target genes c-JUN and MEF2C. The overexpression of circSVIL in chicken myoblasts showed that it promoted myogenic differentiation, possibly through sponging miR-203 (Ouyang, 2018). Other circRNA such as circFGFR4 and circFUT10 found in bovine also acted as the sponges of miR-107 and miR-133a and were involved in the proliferation and differentiation of myoblasts (Li et al., 2018). 
Also, it is reported that circRNAs have open reading frames which have the capacity of translation (Legnini et al., 2017). An example is the circRNA ZNF609 which has an open reading frame covering from the start codon, in common with the linear transcript, that is stopped at an in-frame stop codon, creating circularization. Circ-ZNF609 can be translated into a specific protein in a splicing-dependent and cap-independent manner, which is involved in muscle cell development (Legnini et al., 2017).

\section{Conclusion and Recommendation}

In whole, circRNA is now an exciting field which is still in its early stages. More thorough studies are required to discover the specific biological and functional roles of circRNAs in muscle, such as muscle exercise and nutrition. The domain of the transcriptome may perhaps be further complex than formerly thought. CircRNAs, as the novel leading of non-coding RNA, would generally be involved in the regulation of biological and physiological developments of skeletal muscles which also is responsible for new possible beneficial targets in meat-type animal genetics and breeding.

\section{Acknowledgments}

This review was supported by the National Science Foundation of China (31672412 and 31972550), the National Science Foundation of Guangdong Province (2017A030307002), the Open Project of Key Lab of Chicken Genetics, Breeding and Reproduction, Ministry of Agriculture and The Key Platform Projects of Innovative Strong School Engineering by Department of Education of Guangdong Province: Efficient Use of Animal Genetic Resources in the Tropics and Healthy Farming (2018302).

\section{Conflicts of interest}

The authors declare no conflicts of interest.

\section{References}

Abdelmohsen K., Panda A.C., De S., Grammatikakis I., Kim J., Ding J., Noh J.H., K i m K.M., Mat t is on J.A., d e Cabo R., Goro s p e M. (2015). Circular RNAs in monkey muscle: age-dependent changes. Aging (Albany. NY), 7: 903-910.

A shwal-Fluss R., Meyer M., P a mudurti N.R., Ivanov A., B artok O., Hanan M., Evanta 1 N., M e m c zak S., R a jew s k y N., K a d e ner S. (2014). CircRNA biogenesis competes with pre-mRNA splicing. Mol. Cell., 56: 55-66.

B a s s e l- D u b y R., O l s o n E.N. (2006). Signaling pathways in skeletal muscle remodeling. Annu. Rev. Biochem.,75: 19-37.

C h e n L.L., Yang L. (2015). Regulation of circRNA biogenesis. RNA Biol., 12: 381-388.

C onw a y A. (2018). World poultry production at nearly 123 million tons in 2018. Poultry Trends, 6.

Du W.W., Yang W., Liu E., Yang Z., Dhaliwal P., Yang B.B. (2016). Foxo3 circular RNA retards cell cycle progression via forming ternary complexes with p21 and CDK2. Nucleic Acids Res., 44: 2846-2858. 
Ivanov A., Memczak S., Wyler E., Torti F., Porath H.T., Orejuela M.R., Piechot ta M., Levanon E.Y., Landthaler M., Dieterich C., Rajewsky N. (2015). Analysis of intron sequences reveals hallmarks of circular RNA biogenesis in animals. Cell Rep., 10: $170-177$.

Kulcheski F.R., Chris t off A.P., Marg is R. (2016). Circular RNAs are miRNA sponges and can be used as a new class of biomarker. J. Biotechnol., 238: 42-51.

L a s d a E., P a r ke r R. (2014). Circular RNAs: diversity of form and function. RNA, 20: 1829-1842.

Legnini I., Di Timoteo G., Rossi F., Morlando M., Briganti F., Sthandier O., Fatic a A., S antini T., Andronache A., Wade M., Laneve P., Rajew s ky N., B ozz o n i I. (2017). Circ-ZNF609 is a circular RNA that can be translated and functions in myogenesis. Mol. Cell., 66: 22-37.

Li C., Li X., Ma Q., Zhang X., Cao Y., Yao Y., You S., Wang D., Quan R., Hou X., Liu Z., Zh a n Q., Li u L., Zh ang M., Yu S., N i W., H u S. (2017). Genome-wide analysis of circular RNAs in prenatal and postnatal muscle of sheep. Oncotarget, 8: 97165-97177.

Li H., We i X., Yang J., Dong D., Ha o D., Huang Y., Lan X., Plath M., Lei C., Ma Y., L in F., B a i Y., Ch en H. (2018 a). circFGFR4 promotes differentiation of myoblasts via binding miR-107 to relieve its inhibition of wnt3a. Mol. Ther. Nucleic Acids., 11: 272-283.

Li H., Yang J., We i X., S ong C., Dong D., Huang Y., Lan X., Plath M., Le i C., Ma Y., Q i X., B a i Y., Chen H. (2018 b). CircFUT10 reduces proliferation and facilitates differentiation of myoblasts by sponging miR-133a. J. Cell. Physiol., 233: 4643-4651.

L i a n g G., Yang Y., N i u G., Ta n g Z., L i K. (2017). Genome-wide profiling of Sus scrofa circular RNAs across nine organs and three developmental stages. DNA Res., 24: 523-535.

Memczak S., Jens M., Elefsinioti A., Torti F., Krueger J., Rybak A., Maier L., Mackowiak S.D., Gregersen L.H., Munschauer M., Loewer A., Zi ebold U., Landthaler M., Kocks C., Le Noble F., Rajewsky N. (2013). Circular RNAs are a large class of animal RNAs with regulatory potency. Nature, 495: 333-338.

N i e M., D e n g Z.L., L i u J., Wang D.Z. (2015). Noncoding RNAs, emerging regulators of skeletal muscle development and diseases. Biomed Res. Int., 2015, 17.

Nits che A., Doose G., Tafer H., Robinson M., S aha N.R., Gerdol M., Can a pa A., H o ffmann S., A m e mi y a C.T., S t a d l e r P.F. (2014). Atypical RNAs in the coelacanth transcriptome. J. Exp. Zool. Part B Mol. Dev. Evol., 322: 342-351.

O u y ang H., Chen X., Wang Z., Yu J., Ji a X., Li Z., L u o W., A b d a 11 a B.A., J eb e s s a E., N i e Q., Zhang X. (2017). Circular RNAs are abundant and dynamically expressed during embryonic muscle development in chickens. DNA Res., 25: 71-86.

O u y an g H., Ch e n X., L i W., L i Z., N i e Q., Z h an g X. (2018). Circular RNA circSVIL promotes myoblast proliferation and differentiation by sponging miR-203 in chicken. Front. Genet., 9: 1-10.

Rybak - Wolf A., S t o t t m e is t e r C., Glaža r P., Jens M., P in o N., H an a n M., B e h m M., B artok O., A shwal-Flus s R., Herzog M., S chreyer L., P a pavasileiou P., Ivanov A., Öhman M., Re fojo D., Ka dener S., Rajewsky N. (2014). Circular RNAs in the mammalian brain are highly abundant, conserved, and dynamically expressed. Mol. Cell, 58: $870-885$.

S a 1 z m a n J., Chen R.E., O l s en M.N., Wang P.L., B rown P.O. (2013). Cell-type specific features of circular RNA expression. PLoS Genet., 9: 1003777.

S chi a ffi no S., S andri M., Murg i a M. (2007). Activity-dependent signaling pathways controlling muscle diversity and plasticity. Physiology, 22: 269-278.

S chi a ffi no S., D y a r K.A., C i c ili o t S., B l a a u w B., S a n d ri M. (2013). Mechanisms regulating skeletal muscle growth and atrophy. FEBS J., 280: 4294-4314.

S he n Y., G u o X., Wang W. (2017). Identification and characterization of circular RNAs in zebrafish. FEBS letters, 591: 213-220.

Sun J., Xi e M., Huang Z., Li H., Chen T., Sun R., Wang J., Xi Q., Wu T., Zhang Y. (2017). Integrated analysis of non-coding RNA and mRNA expression profiles of 2 pig breeds differing in muscle traits. J. Anim. Sci., 95: 1092-1103.

Venø M.T., H a n s en T.B., Venø S.T., C l a u s e n B.H., Grebing M., F in s en B., Holm I.E., K j e m s J. (2015). Spatio-temporal regulation of circular RNA expression during porcine embryonic brain development. Genome Biol., 16: 245. 
Vic en s Q., We st th of E. (2014). Previews Biogenesis of Circular RNAs. Cell, 159: 13-14.

We i X., Li H., Yang J., H a o D., D ong D., Huang Y., L an X., Plath M., Le i C., L in F., B a i Y., Chen H. (2017). Circular RNA profiling reveals an abundant circLMO7 that regulates myoblasts differentiation and survival by sponging miR-378a-3p. Cell Death Dis., 8: e3153.

Westholm J.O., Miura P., Olson S., Shenker S., Joseph B., Sanfilippo P., Celniker S.E., Gravele y B.R., La i E.C. (2014). Genome-wide analysis of drosophila circular RNAs reveals their structural and sequence properties and age-dependent neural accumulation. Cell Rep., 9: 1966-1981.

Received: 6 V 2019

Accepted: 6 VIII 2019 